\title{
La isla de calor urbana del área metropolitana de Santiago (AMS) de Chile a partir de diferencias térmicas de los observatorios de Talagante y Cerrillos
}

The urban heat island effect of the Santiago Metropolitan Area (SMA) based on thermal differences between two observatories

\author{
Pablo Sarricolea Espinoza ${ }^{1}$ \\ Javier Martín Vide $^{2}$
}

\section{Resumen}

Este artículo presenta el análisis de la intensidad de la isla de calor urbana (ICU) de Santiago para el año 2010. El objetivo es conocer la hora en que la ICU se manifiesta con mayor intensidad, y con ello, se modela mediante un conjunto de parámetros meteorológicos. Los resultados indican que la ICU en promedio es más intensa en verano y primavera. El máximo desarrollo de la ICU se consigue a medianoche, con diferencias medias entre $2,8^{\circ} \mathrm{C}$ y $4,8^{\circ} \mathrm{C}$. La máxima anomalía térmica en el orden estacional se alzó por encima de los $9^{\circ} \mathrm{C}$, tanto en verano como en primavera. Las variables explicativas de la ICU fueron la nubosidad, la humedad relativa y la presión atmosférica. Como conclusión, se puede señalar que es posible generar un sistema de pronóstico de la ICU mediante la obtención de los valores de humedad relativa, la presión atmosférica y la nubosidad, desde las predicciones sinópticas y mesoescalares.

Palabras clave: isla de calor urbana, modelos de regresión múltiple, Santiago de Chile.

1 Doctor en Geografía, Universidad de Barcelona, España. Profesor Asistente del Departamento de Geografía de la Universidad de Chile. Chile.psarricolea@uchilefau.cl

2 Doctor en Geografía, Universidad de Barcelona, España. Catedrático de Universidad, Departamento de Geografía Física y A.G.R., Grupo de Climatología, Universidad de Barcelona. España. jmartinvide@ub.edu 


\section{Abstract}

This study analyses the intensity of the urban heat island (UHI) in Santiago of Chile in 2010. The objective is to identify the hour in which the UHI presents its highest intensity. The intensity of the urban heat island is modelled using series of meteorological parameters. The results indicate that the urban heat island is on average more intense in summer and spring. The highest urban heat island intensity is observed during midnight. The variables used to evaluate urban heat island intensity are: cloudiness, relative humidity, and atmospheric pressure. Cloudiness adds up to $1^{\circ} \mathrm{C}$ to the UHI while a $50 \%$ increase in relative humidity reduces $\mathrm{UHI}$ by more than $3^{\circ} \mathrm{C}$. Atmospheric pressure has a strong positive influence on UHI. In conclusion, it is worth pointing out that these three variables can generally be forecasted at least 180 hours in advance meaning the greatest UHI events could be predicted and prevented.

Keywords: multiple regression models, Santiago of Chile, urban heat island. 


\section{Introducción}

La intensidad de la isla de calor urbana (ICU) es un fenómeno que se ha estudiado de diversas maneras en función del desarrollo de la técnica. No obstante la diversidad de herramientas y técnicas que se han publicado, en especial a partir del último tercio del siglo $\mathrm{xx}$, no son sustitutorias de los "métodos tradicionales", sino más bien complementarias. En este sentido, una estrategia aún vigente para establecer la intensidad de la isla de calor urbana corresponde a la estimación de las diferencias termométricas de al menos dos observatorios, uno rural y otro urbano, los cuales deben cumplir las condiciones descritas por Lowry (1977), referidas a que los observatorios posean una localización comparable en términos geográficos y de climatología regional, para así estimar el efecto urbano.

Las reglas establecidas por Lowry (1977) corresponden a condiciones mínimas en los estudios de la ICU, y particularmente, permiten establecer el efecto urbano, y así conocer la máxima intensidad de ella. Cabe destacar que, recientemente, Memon et al. (2008) han realizado una compilación de las últimas investigaciones de ICU. En dicho trabajo se recogen, entre otros, los estudios efectuados con observatorios meteorológicos, destacando los casos de Fairbanks en Alaska, la cual presenta intensidades de hasta $1^{\circ} \mathrm{C}$ (Magee et al., 1999), de Korea con $3,4{ }^{\circ} \mathrm{C}$ (Kim \& Baik, 2005), de ciudad de México y New York con más de $5{ }^{\circ} \mathrm{C}$ (Jáuregui, 1997; Gedzelman et al., 2003), Hong
Kong con $10,5{ }^{\circ} \mathrm{C}$ (Memon et al., 2008) y Lódź con $12{ }^{\circ} \mathrm{C}$ (Klysik y Fortuniak, 1999). El único caso que ofrece intensidades negativas de la ICU es la ciudad de Granada, Nicaragua, con -2 ${ }^{\circ} \mathrm{C}$ (Montávez et al., 2000).

Además de establecer la máxima intensidad de la isla de calor urbana (MIICU3), es muy importante conocer la época del año en que logra este máximo. No obstante, las diferentes investigaciones hechas hasta ahora no consiguen consenso. En su mayoría, los autores señalan el invierno como la estación donde la ICU es mayor, debido al uso de calefacción y, por ende, aporte de calor antropogénico al balance radiativo (Moreno, 1993; Montávez et al., 2000; Liu et al., 2007). Pero algunos indican que la ICU es más intensa en verano (Eliasson, 1994; Klysik \& Fortuniak, 1999; Kolokotroni \& Giridharan, 2008) y en otoño (Alonso et al., 2003). También ciertas ciudades presentan biestacionalidad de la máxima intensidad de la ICU: estaciones de otoño-verano (Yagüe et al., 1991; Gedzelman et al., 2003) y otoño-invierno (Kim \& Baik, 2002). Esto demuestra que es muy difícil establecer una pauta global respecto a la estacionalidad de la máxima intensidad de la isla de calor, y que ella dependerá de las condiciones propias de la localización geográfica del emplazamiento de la ciudad, su entorno y su clima (Sarricolea et al., 2008).

3 Típicamente, la máxima intensidad de la isla de calor se manifiesta después de la puesta de sol, con cielo despejado y vientos débiles. 
Para la ciudad de Santiago de Chile, los primeros estudios de la ICU fueron llevados a cabo por Aceituno y Ulriksen (1981) y luego por Salinas (1982), todos ellos parte de la misma investigación, que, realizando transectos térmicos nocturnos con automóvil, detectaron diferencias de $3{ }^{\circ} \mathrm{C}$ a $4{ }^{\circ} \mathrm{C}$ e incluso de más de $10{ }^{\circ} \mathrm{C}$ entre el centro y el entorno suburbano. Dos décadas más tarde se hicieron nuevos transectos por otro equipo de investigadores de la Universidad de Chile, y los resultados obtenidos por Molina (2007) fueron similares a los de décadas pasadas, pero esta vez se realizaron transectos en tres momentos del día (10:30, 14:30 y 22:30 horas), y se correlacionaron con usos de suelo. No obstante, ninguna investigación efectuada para el área metropolitana de Santiago de Chile (AMS, en adelante) ha establecido la máxima intensidad del fenómeno, tanto en mes, hora, estación del año y condición sinóptica. Por lo tanto, este trabajo tiene como propósito dar respuesta, con la información de dos observatorios, a dichos interrogantes. Además, se espera construir un modelo que explique mediante variables meteorológicas la mayor intensidad de la isla de calor. Las hipótesis de trabajo por lo tanto serán:

1. La isla de calor urbana tendrá una mayor magnitud unas pocas horas después de la puesta de sol, y en aquellos días de calma, cielo despejado y circulación anticiclónica.

2. La isla de calor urbana del aire de Santiago debería alcanzar, según su población, una máxima intensidad comprendida entre $9,6{ }^{\circ} \mathrm{C}$ y $13,6{ }^{\circ} \mathrm{C}$, dependiendo de su morfología urbana y el tipo de tiempo de Jenkinson y Collison (J\&C).

3. Las variables meteorológicas que mejor explican la isla de calor urbana del aire (ICU) deberán ser la intensidad del viento, la nubosidad, la presión atmosférica, la humedad relativa, $\mathrm{y}$, en segundo lugar, las horas de sol y el día de la semana.

\section{Materiales y métodos}

Se han obtenido los datos oficiales de los observatorios de Cerrillos y Talagante para el año 2010. Ambas estaciones pertenecen al sistema nacional de información de calidad de aire del Gobierno de Chile. Se considera que dichos observatorios son los que mejor se adecúan a los criterios de Lowry (1977). Además, los datos poseen una resolución horaria de las temperaturas, por lo cual permiten establecer la máxima intensidad de la isla de calor.

Metodológicamente, la consideración de sólo un año no es común en climatología regional o zonal, pues para ella, es recomendable una serie de treinta años. No obstante, en estudios de climatología urbana se admite que un año posee significación climática, pues el efecto urbano es un fenómeno de carácter estructural y no coyuntural (Moreno, 1993; Lee et al., 2012). Además, tampoco se pudo contar con 
una serie larga, pues el observatorio de Talagante sólo está vigente desde fi- nales de 2009. La Figura 1 muestra la localización de ambas estaciones.

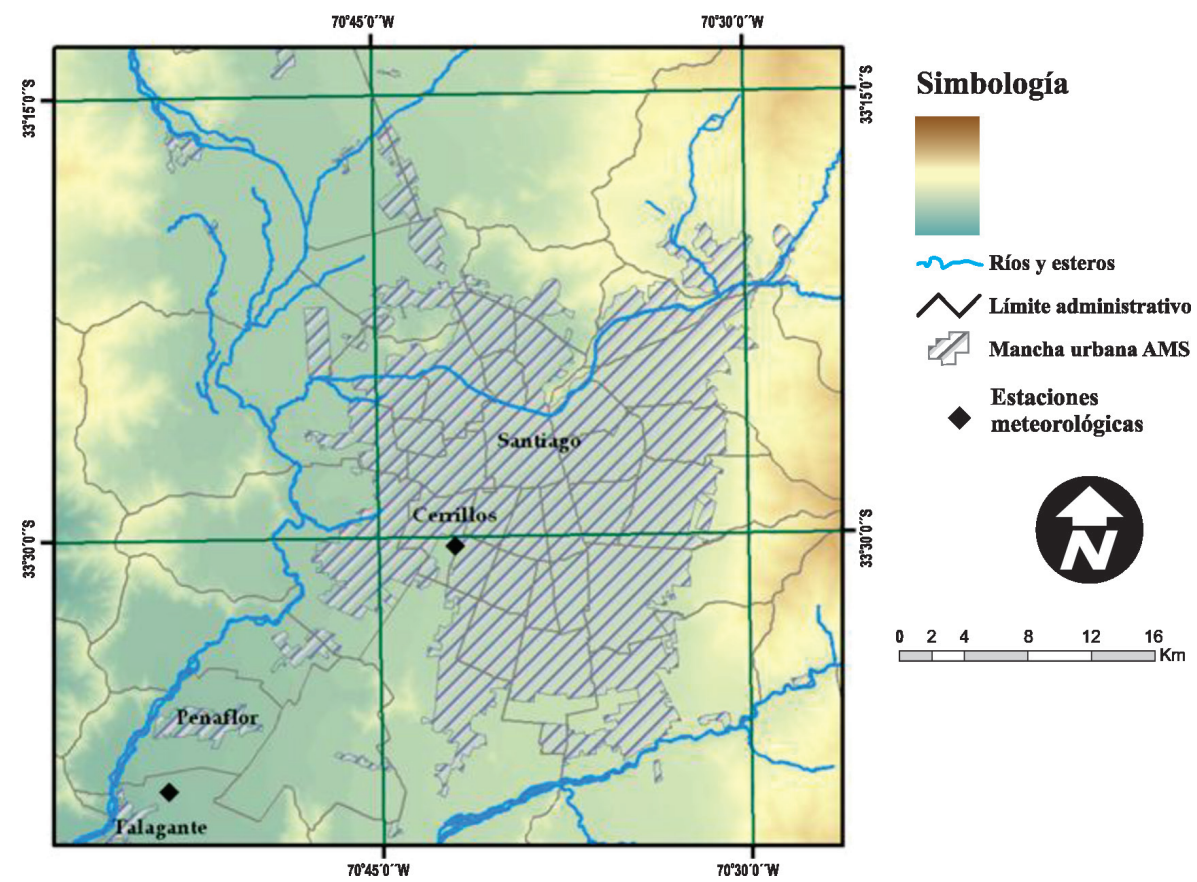

Figura 1. Mapa de localización de las estaciones meteorológicas.

La estación de Cerrillos y Talagante están a una altitud de 540 y $430 \mathrm{msnm}$ respectivamente, y distan entre sí un total de $24 \mathrm{~km}$. Ambas estaciones representan las condiciones del área metropolitana y del entorno rural de Santiago, respectivamente. En total se procesaron 8.760 pares de datos, y para estimar la intensidad de la isla de calor se utilizó la siguiente expresión:

$$
\Delta t=t_{U}-t_{R}
$$

Donde $\Delta t$ representa la intensidad de la isla de calor urbana para distintas horas del día, siendo $t_{U}$ la temperatura medida en la estación meteorológica de Cerrillos, y $t_{R}$ la correspondiente temperatura registrada en la estación de Talagante. El intervalo de medición es horario, y a partir de ellos se ha calculado la variación diaria, mensual y estacional. Para determinar la máxima intensidad de la ICU, se seleccionaron los máximos valores de en el año 2010, para cada mes y estación, además de la franja horaria donde las diferencias fueron más importantes. Con el propósito de categorizar las intensidades de la ICU, se utilizó la propuesta de Fernández (1996) aplicada a la ciudad de Madrid, es decir: 
- ICU débil, que es aquella que no supera $\operatorname{los} 2{ }^{\circ} \mathrm{C}$,

- ICU moderada, que registra entre 2 y $4^{\circ} \mathrm{C}$,

- ICU fuerte, que alcanza entre 4 y 6 ${ }^{\circ} \mathrm{C}, \mathrm{y}$

- ICU muy fuerte, la que sobrepasa los $6^{\circ} \mathrm{C}$.

Cabe destacar que la información clasificada fue luego analizada en función de los tipos de tiempo, calculados mediante el método automático de Jenkinson y Collison. Con ello, fue posible conocer la relación entre los tipos de tiempo y las intensidades de la isla de calor. La clasificación de Jenkinson y Collison fue obtenida a partir de Sarricolea et al. (2011), que realizaron la clasificación de todos los días en Chile central desde 1950 hasta 2010.

Para la modelación de la ICU se optó por incorporar las variables de día de la semana con valores de 0 a 6 ( 0 representa al domingo, 1 al día lunes y así hasta el sábado con un número 6), nubosidad en Octas ( 0 a 8), humedad relativa del aire (en porcentaje), velocidad del viento $(\mathrm{m} / \mathrm{s})$, horas de sol (horas día) y presión atmosférica ( $\mathrm{hPa}$ reducidos al nivel del mar), tal como se muestra en la Figura 2. Además se modeló la ICU para cada una de las estaciones del año.

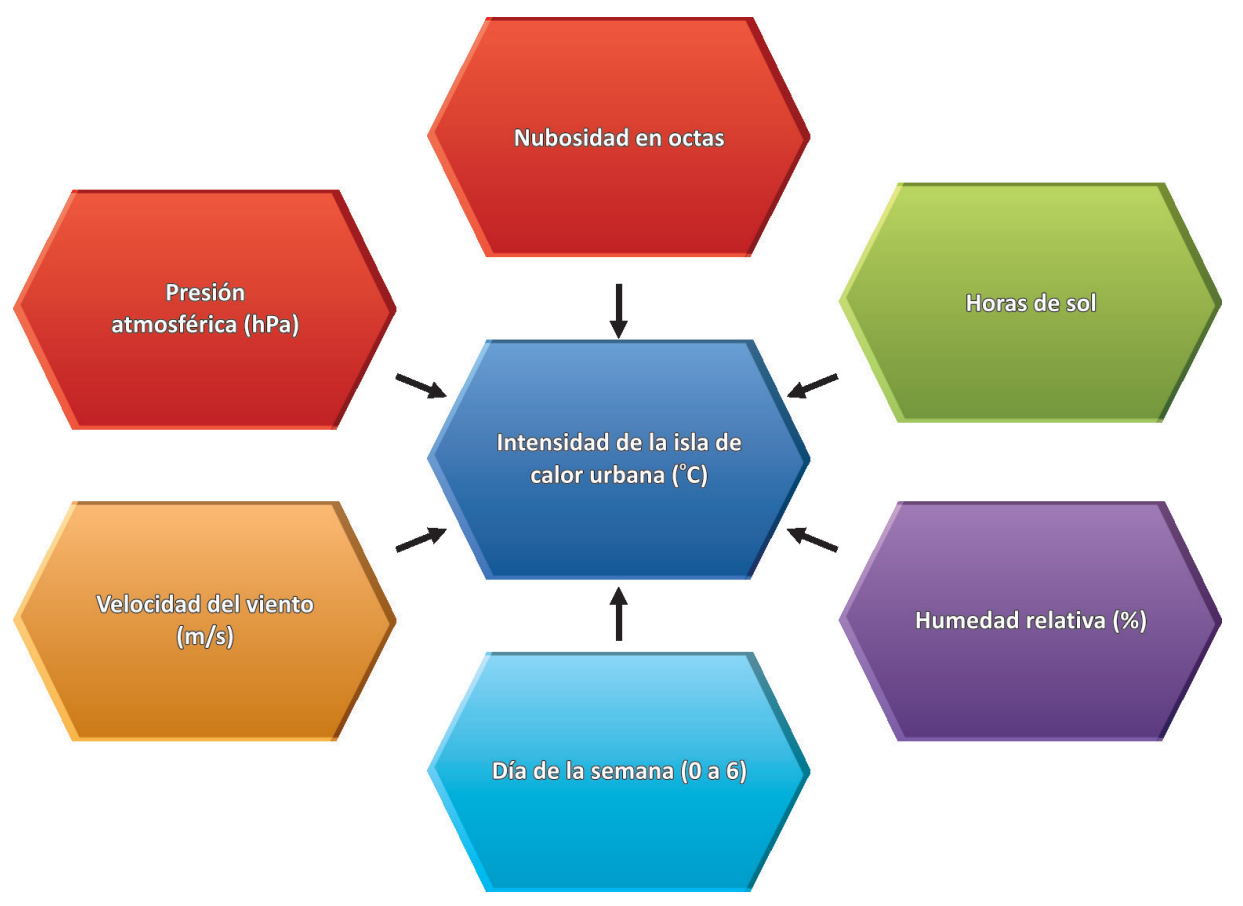

Figura 2. Variables utilizadas para la modelación de la isla de calor urbana del AMS. 


\section{Resultados}

En primer lugar se analizó si Cerrillos y Talagante son comparables desde el punto de vista estadístico. La Tabla 1 muestra que, en efecto, los valores son significativamente diferentes en cuanto a sus valores de tendencia central, variabilidad y distribución (de asimetría positiva y de forma mesocúrtica). Cabe destacar que las temperaturas mínimas urbanas son mayores a las rurales en casi $2{ }^{\circ} \mathrm{C}$, mientras que las máximas temperaturas se alcanzan en las áreas rurales. Esto demuestra que la ciudad posee menor amplitud térmica por el efecto de isla de calor, lo cual queda reflejado en los $6{ }^{\circ} \mathrm{C}$ de diferencia del rango. Adicionalmente, el promedio térmico de la ciudad es casi $3{ }^{\circ} \mathrm{C}$ más alto que en el área rural, lo cual posee fuertes repercusiones en la suma de grados día.

Tabla 1. Resumen estadístico anual de la temperatura de las estaciones de Cerrillos y Talagante.

\begin{tabular}{lcc}
\hline Resumen estadístico & Cerrillos & Talagante \\
\hline Media $\left({ }^{\circ} \mathrm{C}\right)$ & 15,96 & 13,19 \\
\hline Varianza & 46,36 & 52,43 \\
\hline Desviación típica & 6,81 & 7,24 \\
\hline Mínimo $\left({ }^{\circ} \mathrm{C}\right)$ & 0,73 & $-3,33$ \\
\hline Máximo $\left({ }^{\circ} \mathrm{C}\right)$ & 35,49 & 37,58 \\
\hline Rango & 34,76 & 40,91 \\
\hline Asimetría tipificada & 13,54 & 21,67 \\
\hline Asimetría & 0,36 & 0,58 \\
\hline Curtosis & $-0,54$ & $-0,28$ \\
\hline Curtosis tipificada & $-10,12$ & $-5,23$ \\
\hline
\end{tabular}

Fuente: elaboración propia.

En la Figura 3 se muestra que la temperatura media de Talagante es menor que en Cerrillos. No obstante, las temperaturas mínimas y máximas son más extremas en Talagante. Ello se podría atribuir al efecto isla de calor en Cerrillos, pues se aprecia una disminución de la amplitud térmica absoluta.

Se calculó $\Delta t=t_{U}-t_{R}$ para analizar el comportamiento de las diferencias de temperatura entre Cerrillos y Talagante. En primer lugar, la isla de calor urbana promedio (Figura 4) es mayor en la medianoche (0:00 horas) y más intensa en el verano, con diferencias medias de 4,8 ${ }^{\circ} \mathrm{C}$, seguida de primavera con $4,4{ }^{\circ} \mathrm{C}$, otoño con $3,8^{\circ} \mathrm{C}$ y finalmente invierno con $2,8{ }^{\circ} \mathrm{C}$. Además, la diferencia de temperatura disminuye en el período diurno hasta ser negativa, pero sólo en primavera (con $-0,3{ }^{\circ} \mathrm{C}$ ), y a las 13:00 


\section{Densidades suavizadas}

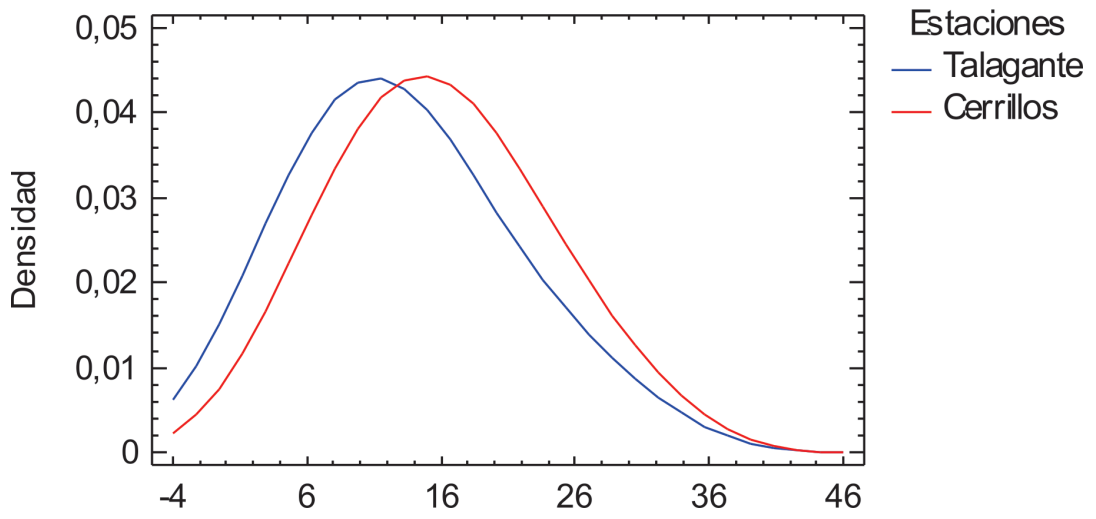

Figura 3. Densidades suavizadas ${ }^{4}$ de las temperaturas medias diarias de Talagante y Cerrillos durante el año 2010.

horas. Visto de otro modo, la ciudad de Santiago pasado el mediodía comienza a calentarse respecto a su entorno rural, alcanzando la máxima diferencia a medianoche; después las diferencias comienzan a disminuir. Dicho ciclo diario da origen a la isla de calor urbana nocturna.

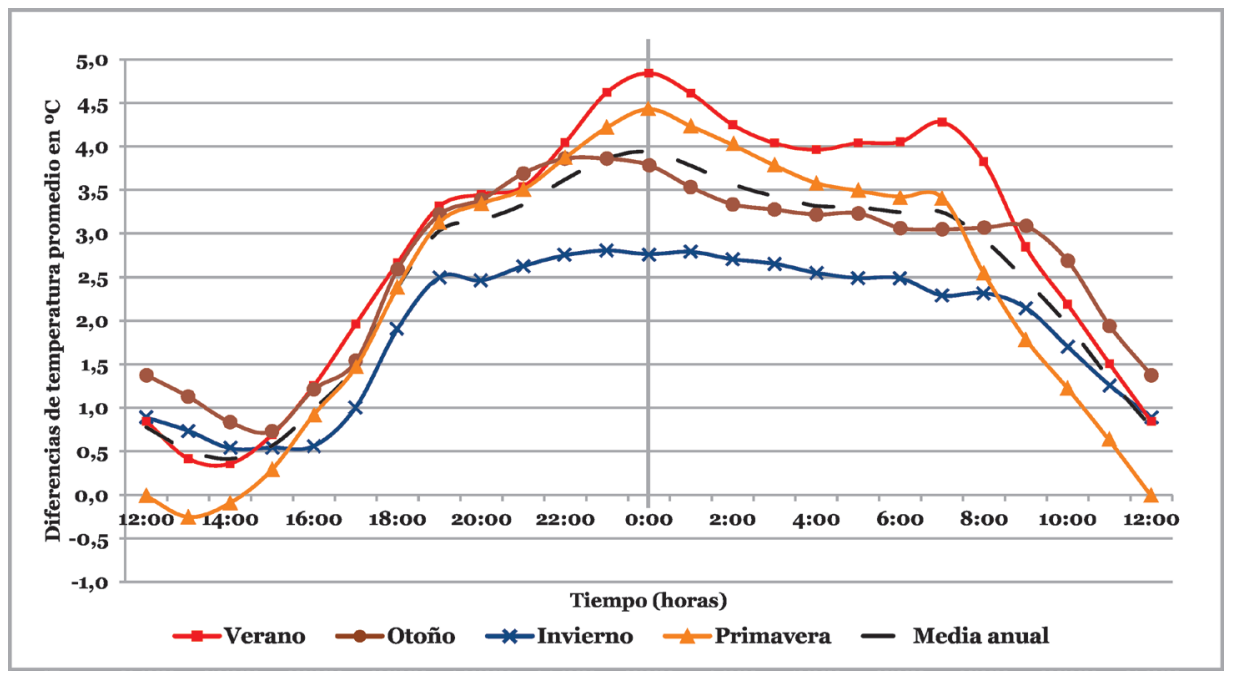

Figura 4. Intensidad promedio de la isla de calor de AMS según estaciones del año 2010.

4 La densidad en un histograma indica la distribución del área bajo la función que en suma representa el total (1 0 $100 \%)$. 
Como se aprecia en la Figura 4, la máxima isla de calor se alcanza entre las 22:00 y las 01:00 horas. Se decide también graficar las diferencias máximas absolutas de temperatura, y así conocer la máxima intensidad de la ICU del área metropolitana de Santiago. En la Figura
5 se observa que los valores absolutos más elevados corresponden a la primavera y el verano $\left(9,6{ }^{\circ} \mathrm{C}\right.$ y $\left.9,4{ }^{\circ} \mathrm{C}\right)$, seguida de otoño $\left(8,5^{\circ} \mathrm{C}\right)$. En el invierno, la MIICU se observa a las 23:00 horas $\left(7,3^{\circ} \mathrm{C}\right)$.

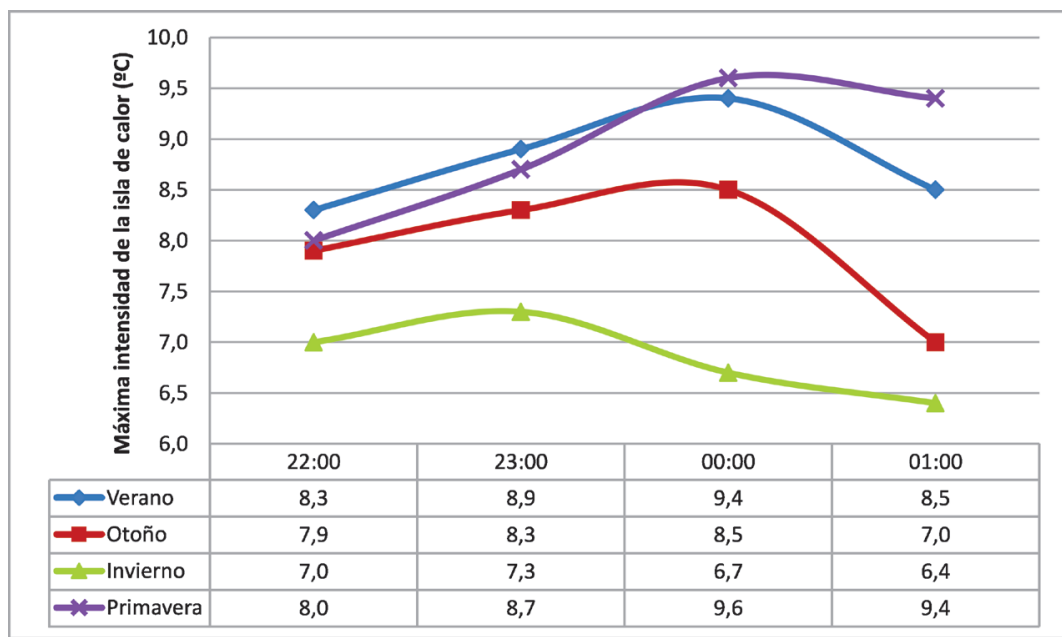

Figura 5. Máxima intensidad de la isla de calor de AMS según estaciones del año 2010.

Respecto a la máxima intensidad de la hicieron pruebas con la distribución isla de calor, se estableció la distribu- Normal, Log-normal, Gamma, Erlang ción que mejor ajusta a la muestra. Se y Weibull (Figura 6). Los test de hipó-

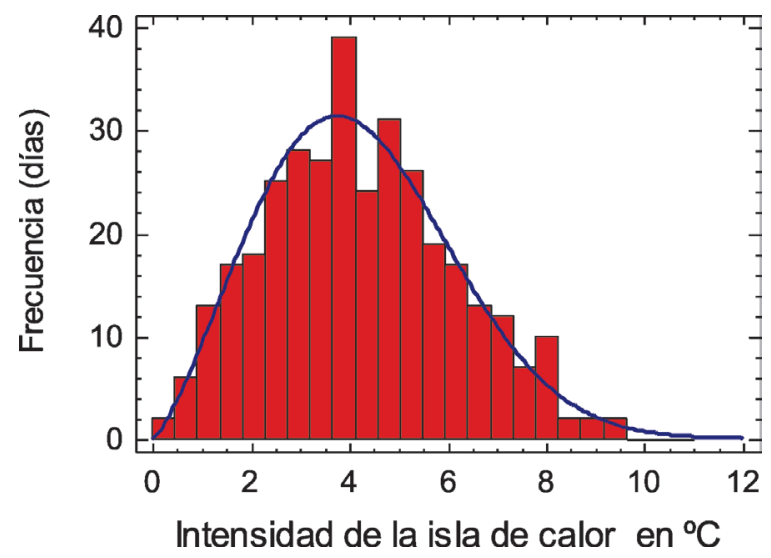

Figura 6. Histograma de la intensidad de la ICU para el año 2010. 
tesis que fueron calculados demuestran que la distribución que mejor ajusta a los valores de temperatura de la isla de calor es la Weibull, con parámetros de forma y escala de 2,4 y 4,8 respectivamente. El test de Kolmogorov-Smirnov indica una distancia máxima de 0,0259 entre la distribución acumulada de los valores de la ICU y la función de distribución. Dado que $p$-value más pequeño de los tests realizados es inferior o igual a 0,10 , se determina que a la ICU le corresponde una distribución Weibull con un nivel de confianza de un $90 \%$.
Las intensidades de la isla de calor, según las categorías de Fernández (1996), se grafican en la Figura 7. Los meses de verano son los que agrupan más días de intensidades muy fuertes, destacándose el mes de enero con catorce días. Se puede señalar que la distribución sumada de días de intensidad débil y moderada es unimodal y con máximos en los meses de junio y agosto, mientras que los días con situaciones de isla de calor muy fuerte y fuerte, poseen la misma distribución, pero centrada en verano.

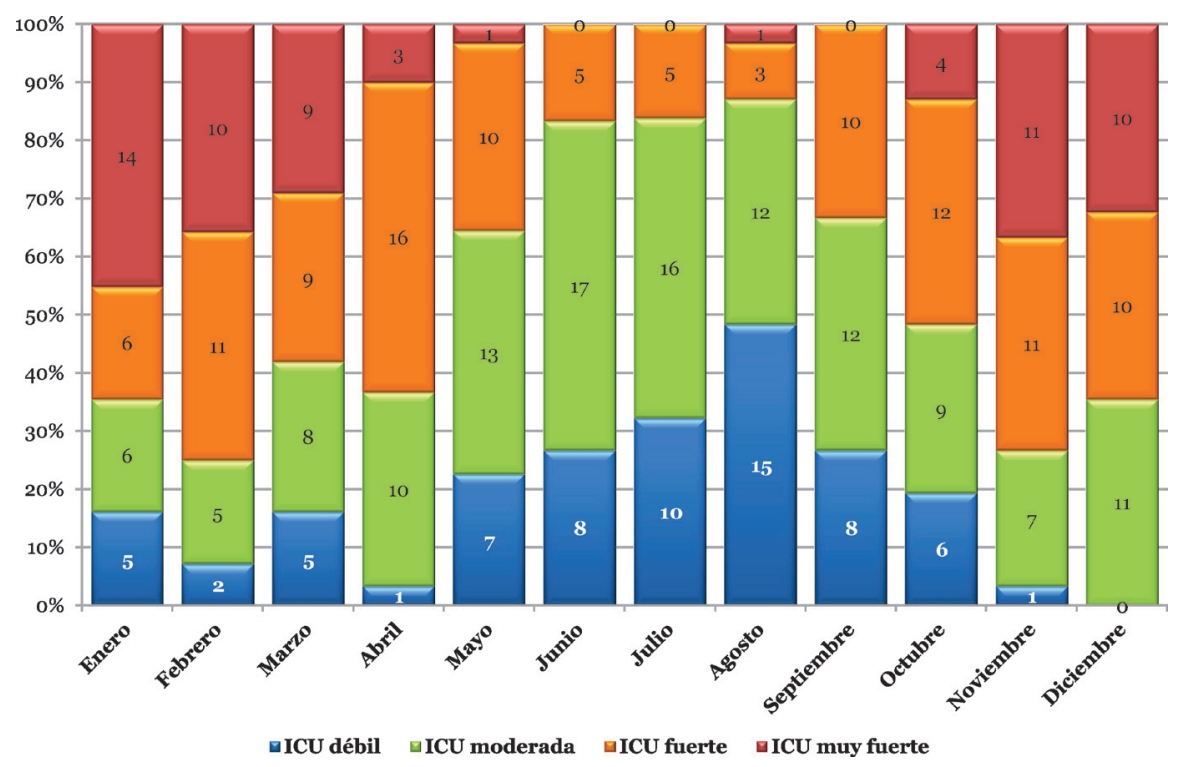

Figura 7. Número y porcentaje de días según meses y categorías de isla de calor urbana del año 2010

De la figura anterior se contabilizan en total, sesenta y tres días, en los que se alcanzan intensidades superiores a $6{ }^{\circ} \mathrm{C}$. De ellos, treinta y cuatro se presentaron en verano, veinte en primavera, ocho en otoño y uno en invierno, tal como muestran la Tabla 2 y la Figura 8a.
Respecto a las configuraciones sinópticas obtenidas desde $\mathrm{J} \& \mathrm{C}$, se observa que para los días de isla de calor urbana muy fuerte (ver Tabla 2 y Figura $8 b$ ), existe un predominio de las situaciones anticiclónicas y con advección de viento del sur y sureste, sumando un $87 \%$ de 
los casos; mientras que los días C, CSE y AW alcanzan una escasa representación.

\begin{tabular}{|lcc}
\hline Resumen estadístico & Cerrillos & Talagante \\
\hline Media $\left({ }^{\circ} \mathrm{C}\right)$ & 15,96 & 13,19 \\
\hline Varianza & 46,36 & 52,43 \\
\hline Desviación típica & 6,81 & 7,24 \\
\hline Mínimo $\left({ }^{\circ} \mathrm{C}\right)$ & 0,73 & $-3,33$ \\
\hline Máximo $\left({ }^{\circ} \mathrm{C}\right)$ & 35,49 & 37,58 \\
\hline Rango & 34,76 & 40,91 \\
\hline Asimetría tipificada & 13,54 & 21,67 \\
\hline Asimetría & 0,36 & 0,58 \\
\hline Curtosis & $-0,54$ & $-0,28$ \\
\hline Curtosis tipificada & $-10,12$ & $-5,23$ \\
\hline
\end{tabular}

Tabla 2. Días en que la ICU supera $6{ }^{\circ} \mathrm{C}$, según estación del año y tipos sinópticos de Jenkinson y Collison (J\&C)3
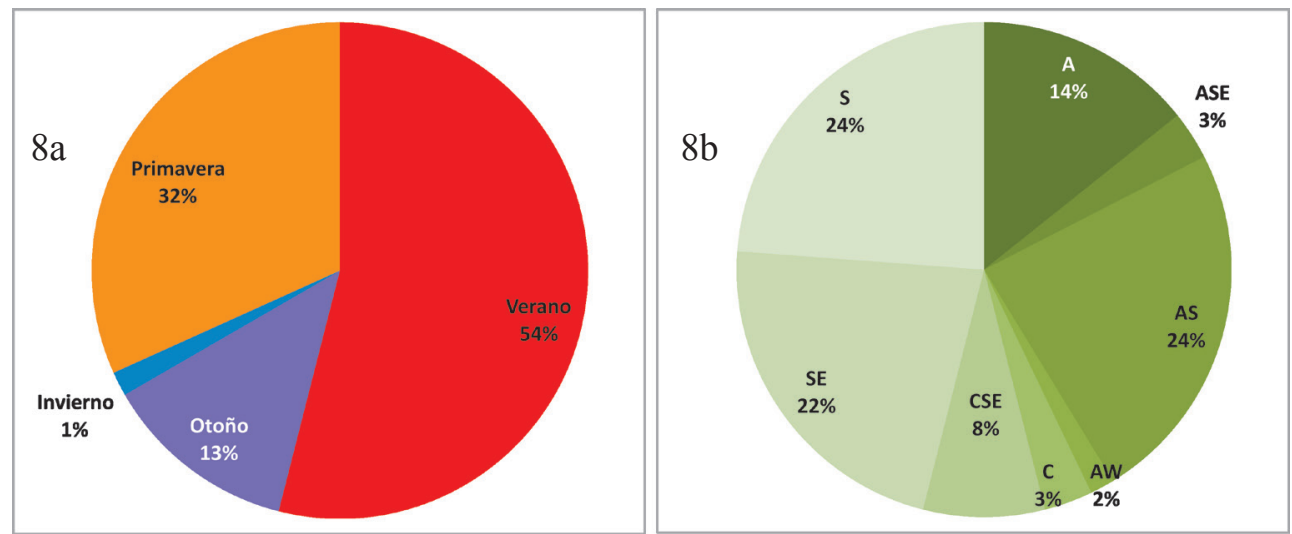

Figura 8. Total porcentual en que la ICU supera $6{ }^{\circ} \mathrm{C}$ según estación del año (izquierda) y tipos Jenkinson

Fuente: elaboración propia.

Ulteriormente, se hizo la modelación de la isla de calor urbana del AMS considerando las variables señaladas en la metodología, que se detallan a continuación: nubosidad en octas, presión atmosférica, humedad relativa del aire, velocidad del viento, horas de sol y día de la semana. Ellas fueron correla-

cionadas con la temperatura de cada uno de los días del año 2010 mediante un modelo de regresión múltiple por pasos sucesivos y de eliminación progresiva. Se realizó un modelo general y uno para cada estación climática: verano, otoño, invierno y primavera. 
Al aplicar el modelo general para todo el año, se aprecia que las variables estadísticamente significativas en el modelo de regresión múltiple, con un p-value menor de 0,05, es decir, significativas con un nivel de confianza del $95 \%$, son la nubosidad, la humedad relativa y la presión atmosférica.

El estadístico $\mathrm{R}^{2}$ indica que el modelo ajustado explica un $61,05 \%$ de la varianza de la intensidad de la isla de calor urbana. El $\mathrm{R}^{2}$ ajustado, que es más apropiado para comparar modelos con diferente número de variables independientes, es de un 60,43\%, es decir, indica un coeficiente de correlación de Pearson de 0,78. El error estándar de la ecuación final para la estimación de la ICU muestra que la desviación estándar de los residuos es de sólo un 1,11. El error absoluto medio (MAE) es de 0,90 , y se corresponde con el valor promedio de los residuos. El estadístico de Durbin-Watson $(\mathrm{DW})^{5}$ indica que dado que el $p$-value es menor que 0,05 , no hay indicios de una autocorrelación serial en los residuos, y ello, con un nivel de confianza del $95 \%$. En la Tabla 3 se observa la estimación de la ecuación final.

Tabla 3. Resultado del modelo general de regresión múltiple de la IICU

\begin{tabular}{lcccc}
\hline \multicolumn{1}{c}{ Variables } & $\begin{array}{c}\text { Estimación } \\
\text { de la } \\
\text { ecuación }\end{array}$ & $\begin{array}{c}\text { Error } \\
\text { estándar }\end{array}$ & $\begin{array}{c}\text { Estadístico } \\
\mathrm{T}\end{array}$ & p-value \\
\hline Constante & $-36,5007$ & 15,742 & $-2,3187$ & 0,02 \\
Nubosidad & $-0,0667$ & 0,0399 & $-1,6701$ & 0,04 \\
\hline Humedad relativa & $-0,0938$ & 0,0069 & $-13,4674$ & 0,00 \\
$\begin{array}{l}\text { Presión } \\
\text { atmosférica }\end{array}$ & 0,0461 & 0,0156 & 2,9576 & 0,00 \\
\hline
\end{tabular}

Fuente: elaboración propia.

Para determinar si el modelo podía ser simplificado, se examinó la Tabla 3. En ella se muestra que el p-value más alto de las variables independientes es de 0,04 , y corresponde a la nubosidad. Puesto que el $p$-value es menor que
0,05 , ese término es estadísticamente significativo con un nivel de confianza del $95 \%$. Consecuentemente, no se debería eliminar ninguna variable del modelo, que genera la siguiente expresión.

$$
\begin{gathered}
I C U=-36,5007-0,0667 \times \text { Nubosidad }_{\text {octas }}-0,0938 \times \text { Humedad relativa\% }^{\circ} \\
+0,0467 \times \text { Presión atmosférica }{ }_{h P a}
\end{gathered}
$$

5 DW examina los residuos para determinar si hay alguna correlación significativa basada en el orden en el cual se presentan los datos. 
El análisis de las distintas estaciones climáticas sitúa al invierno con el menor $\mathrm{R}^{2}$, alcanzando un $41,33 \%$, mientras que verano y primavera poseen un $\mathrm{R}^{2}$ de $63,55 \%$ y $43,46 \%$, respectivamente.
La estación del año con el mayor valor del coeficiente de determinación es otoño con un $74,01 \%$. A continuación, se presentan las ecuaciones que consideran las tres variables para cada estación.

$$
\begin{aligned}
& I C U_{\text {verano }}=-193,88- 0,0143 \times \text { Nubosidad }_{\text {octas }}-0,1125 \times \text { Humedad relativa\% } \\
&+0,2026 \times \text { Presión atmosférica } \\
& h P a
\end{aligned}
$$

$$
\begin{aligned}
& \text { IC } U_{\text {otoño }}=-103,81- 0,1663 \times \text { Nubosidad }_{\text {octas }}-0,0529 \times \text { Humedad relativa\% } \\
&+0,1096 \times \text { Presión atmosférica } \\
& h P a
\end{aligned}
$$

$$
\begin{aligned}
& I C U_{\text {invierno }}=-56,62- 0,0011 \times \text { Nubosidad }{ }_{\text {octas }}-0,0747 \times \text { Humedad relativa\% } \\
&+ 0,0640 \times \text { Presión atmosférica } \\
& h P a
\end{aligned}
$$

$$
\begin{aligned}
& I C U_{\text {primavera }}=-44,93- 0,0084 \times \text { Nubosidad }_{\text {octas }}-0,0886 \times \text { Humedad relativa\% } \\
&+0,0539 \times \text { Presión atmosférica } \\
& h P a
\end{aligned}
$$

En las ecuaciones anteriores queda clara la relación inversa entre intensidad de la isla de calor y las variables nubosidad y humedad relativa, al igual que la relación positiva entre presión atmosférica e ICU. Con los resultados se puede ver la sensibilidad de la intensidad de la ICU a los cambios en las variables meteorológicas del modelo, que se puede apreciar en la Tabla 4. En dicha tabla se observa que la nubosidad disminuye la IICU anual, tal como en el resto de las estaciones, siendo la estación de otoño la más afectada por el cielo cubierto, mientras que invierno es la estación menos sensible. Así por ejemplo, en otoño un cielo cubierto implica una disminución de $-1,33{ }^{\circ} \mathrm{C}$ y en invierno de sólo $-0,01{ }^{\circ} \mathrm{C}$. La variable más importante y sensible a los cambios es sin duda la humedad relativa, pues su aumento en $50 \%$ implica una disminución de la intensidad de la ICU entre $2,7^{\circ} \mathrm{C}$ y 5,62 ${ }^{\circ} \mathrm{C}$. La presión atmosférica también tiene impactos en la temperatura urbana, sobre todo en verano, pues la acentuación de un anticiclón en $20 \mathrm{hPa}$ implica un aumento de la IICU de hasta $4,05{ }^{\circ} \mathrm{C}$ en verano.

\section{Discusión}

Los resultados indican que la isla de calor de AMS, en promedio, es más intensa en verano. Esto no es atípico, pues hay evidencias en otras ciudades de mayor intensidad de ICU en verano (Elias- 
Tabla 4. Respuesta o sensibilidad de los cambios en las variables de los modelos de la intensidad de la isla de calor urbana del AMS.

\begin{tabular}{|c|c|c|c|c|c|}
\hline $\begin{array}{l}\text { Variables del modelo de } \\
\text { regresión múltiple }\end{array}$ & $\begin{array}{l}\triangle \mathrm{ICU} \text { de } \\
\text { verano en } \\
{ }^{\circ} \mathrm{C}\end{array}$ & $\begin{array}{l}\triangle \mathrm{ICU} \text { de } \\
\text { otoño en } \\
{ }^{\circ} \mathrm{C}\end{array}$ & $\begin{array}{c}\triangle \mathrm{ICU} \text { de } \\
\text { invierno en } \\
{ }^{\circ} \mathrm{C}\end{array}$ & $\begin{array}{c}\triangle \mathrm{ICU} \text { de } \\
\text { primavera en } \\
{ }^{\circ} \mathrm{C}\end{array}$ & $\begin{array}{c}\triangle \mathrm{ICU} \\
\text { anual en } \\
{ }^{\circ} \mathrm{C}\end{array}$ \\
\hline $\begin{array}{l}\text { Cambio desde condición } \\
\text { despejada a cubierto } \\
(0 \text { a } 8 \text { 0ctas })\end{array}$ & $\Sigma^{-0,11}$ & $\sqrt{-1,33}^{-1,33}$ & $\Sigma^{-0,01}$ & $\Sigma^{-0,07}$ & $\sqrt{-0,53}^{-0,53}$ \\
\hline $\begin{array}{l}\text { Aumento de la humedad } \\
\text { relativa en un } 50 \%\end{array}$ & $v^{-5,62}$ & $\nabla^{-2,65}$ & $\nabla^{-3,74}$ & $\nabla^{-4,43}$ & $\nabla^{-4,69}$ \\
\hline $\begin{array}{l}\text { Aumento de la presión } \\
\text { atmosférica en } 20 \mathrm{hPa} \\
\text { (desde } 1013 \mathrm{hPa} \text { ) }\end{array}$ & ن 4,05 & ¿ 2,19 & ن 1,28 & $ن 1,08$ & $\Sigma 0,92$ \\
\hline
\end{tabular}

Fuente: elaboración propia.

son, 1994; Klysik \& Fortuniak, 1999; Kolokotroni \& Giridharan, 2008), e incluso la ciudad de Rancagua, localizada a $87 \mathrm{~km}$ de Santiago de Chile, también tiene al verano como la estación de mayor intensidad de la isla de calor (Sarricolea et al., 2008). En contraparte, es el invierno la estación que presenta una menor magnitud de la isla de calor, lo cual permite definir hipótesis de las causas, por ejemplo:

- La escasa radiación solar en las áreas urbanas en invierno, por causa del entramado de edificios y sombras, y la declinación solar, no permite una suficiente acumulación de calor para ser liberado durante la noche.

- La zona más densamente construida está destinada a usos de oficinas y comercio, por lo cual, la utilización de calefacción en ella durante la noche no es tan alta como en otros sectores residenciales.

- La anulación de la isla de calor por brisas de montaña e inversión térmica de carácter radiativo, lo cual provoca a veces una periferia con temperaturas similares a las de la ciudad durante la noche.

La máxima intensidad de la isla de calor llega a $9,6{ }^{\circ} \mathrm{C}$ en primavera, $9,4{ }^{\circ} \mathrm{C}$ en verano, y $8,5^{\circ} \mathrm{C}$ y $7,3{ }^{\circ} \mathrm{C}$ en otoño e invierno, respectivamente. Según el modelo de Oke (1973), la población de Santiago de Chile (seis millones de habitantes) generaría una ICU entre $9,6{ }^{\circ} \mathrm{C}$ y $13,6{ }^{\circ} \mathrm{C}$, dependiendo de si su morfología urbana es más similar a las ciudades de Europa o de Norteamérica, tal como sugiere la Figura 9, don- 
de se ha situado a Santiago de Chile. Sin duda, los resultados muestran que el AMS posee una isla de calor que se ajusta más a una ciudad europea, lo cual es consistente con el origen de las ciuda- des coloniales de América Latina, pero contradictorio con el actual modelo de ciudad dispersa que se ha instalado en las ciudades globales.
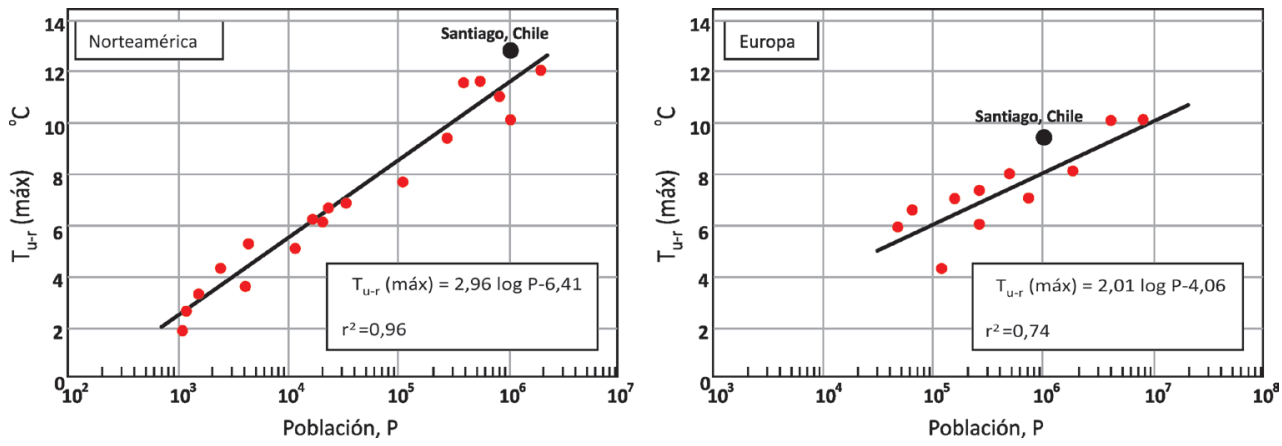

Figura 9. Máxima intensidad pronosticada para la ICU de Santiago según el tipo de ciudad y sus montos de población.

Fuente: elaboración propia con base en Oke (1973).

La clasificación de Fernández (1996) referida a categorías de intensidades de la ICU, confirman al verano como la estación con mayor frecuencia de situaciones de isla de calor que supera el umbral de $6{ }^{\circ} \mathrm{C}$. Y durante el año, los días de ICU muy fuerte están relacionados con situaciones de tiempo anticiclónico y advectivo. No obstante, hay días con una configuración sinóptica de carácter ciclónico que poseen ICU muy fuerte, lo cual merece ser estudiado, a pesar de su escasa representación.

El conocimiento de las variables más influyentes sobre la intensidad de la isla de calor urbana ha sido un asunto de permanente interés de la investigación en climatología urbana, y cada vez se poseen más herramientas estadísticas que permiten llevar a cabo mejores y complejos análisis que consideran un amplio conjunto de variables. Sin embargo, para estudios con dos observatorios, las variables clásicamente utilizadas han sido la presión atmosférica, las configuraciones sinópticas, la nubosidad, el viento y la humedad relativa. Para el caso del AMS se establece que sólo tres de ellas son significativas en explicar estadísticamente la ICU, y con valores del coeficiente de determinación en general moderados $\left(\mathrm{R}^{2}\right.$ entre 41,33 $\%$ y 74,01 \%), pero con $p$-value que permite decir que los valores son significativos al $95 \%$.

\section{Conclusiones}

El análisis de la isla de calor urbana de Santiago mediante dos observatorios meteorológicos durante el año 2010, 
muestra que su máxima intensidad se manifiesta a medianoche (entre las 23:00 y las 00:00 horas), y con valores que sitúan al verano y la primavera como las estaciones de mayor intensidad del fenómeno. A partir de las 16:00 horas, la ciudad aparece claramente más cálida que su entorno rural, aumentando la diferencia hasta las 18:00 horas, y manteniéndose luego hasta las 20:00 horas, para luego seguir aumentando hasta las 23:00 horas en invierno y las 00:00 horas en el resto de las estaciones. En consecuencia, para el AMS la isla de calor se manifiesta más cerca de la medianoche que unas horas después de la puesta de sol. Los días de calma, con cielo despejado y circulación anticiclónica, son los que presentan una mayor intensidad de la isla de calor.

La máxima intensidad de la isla de calor del aire de Santiago es algo menor a la que debería tener, según los montos de población que posee. En efecto, llega a $9,6{ }^{\circ} \mathrm{C}$ en primavera, $9,4{ }^{\circ} \mathrm{C}$ en verano, $8,5{ }^{\circ} \mathrm{C}$ en otoño y $7,3{ }^{\circ} \mathrm{C}$ en invierno. Sin duda, el AMS posee una isla de calor que se asemeja más a una ciudad europea, lo cual es consistente con el origen de las ciudades coloniales de América Latina, pero contradictorio con el actual modelo de ciudad dispersa que se ha instalado en nuestra capital. De seguir esta tendencia de urbanismo disperso, del tipo de Norteamérica, no se descarta una intensificación importante de la magnitud de la isla de calor.

Los tipos de tiempo de J\&C son muy efectivos a la hora de entender y pronosticar la intensidad de la isla de calor.
Según el método, de los sesenta y tres días de ICU muy intensa $\left(>6{ }^{\circ} \mathrm{C}\right)$, un alto porcentaje corresponde a situaciones de circulación anticiclónica. Esto confirma que las situaciones favorables para la ICU son aquellas de carácter anticiclónico. Destacan, además, los días con advección del sur.

Las variables explicativas más relevantes de la ICU en el AMS son la nubosidad, la humedad relativa y la presión atmosférica. La variable viento, pese a quedar excluida del modelo, es de gran importancia en otras ciudades. Sin embargo, puede que se haya subestimado en nuestro caso la importancia del viento al utilizar sólo una estación meteorológica, por lo que se sugiere seguir indagando en ella como variable explicativa.

Los niveles de explicación de la varianza oscilan entre el $41 \%$ en invierno y el $74 \%$ en otoño. Dado que para la estación de invierno los niveles de explicación son bajos, hay que seguir indagando cuáles son las variables que explican mejor la ICU en dicha estación.

El cambio de condición de cielo despejado a cubierto implica una disminución de $1,33{ }^{\circ} \mathrm{C}$ en la intensidad de la isla de calor, mientras que un aumento en un $50 \%$ de la humedad relativa, reduce la ICU en $5,6^{\circ} \mathrm{C}$. Por lo tanto, si se aumenta la humedad relativa del aire mediante áreas verdes, se podría reducir la ICU.

Es posible y recomendable generar un sistema de pronóstico de la intensidad de la isla de calor urbana, a partir de la información de las predicciones sinópti- 
cas y mesoescalares, que proporcionan valores de humedad relativa, presión atmosférica y nubosidad, que han sido las variables seleccionadas por el modelo.
A partir de ellas, se podrá prevenir a la población y anticiparse a problemas ligados a las olas de calor, especialmente en verano.

\section{Literatura citada}

Aceituno, P. \& Ulriksen, P. (1981). Efecto de isla calórica en santiago. Resultados preliminares. Tralka. 2 (1), 39-56.

Alonso, M., Labajo, J. \& Fidalgo, M. (2003). Characteristics of the urban heat island in the city of Salamanca, Spain. Atmósfera, 137-148.

Eliasson, I. (1994). Urban-suburban-rural air temperature differences related to street geometry. Physical Geography (15), 1-22.

Fernández, F. (1996). Manual de climatología aplicada. Madrid, España: Síntesis.

Gedzelman, S., Austin, S., Cermak, R., Stefano, N., Partridge, S., Quesenberry, S., et al. (2003). Mesoscale aspects of the Urban Heat Island around New York City. Theor. Appl. Climatol (75), 29-42.

Jáuregui, E. (1997). Heat island development in Mexico City. Atmospheric Environment, 31, 3821-3831.

Kim, Y. \& Baik, J. (2002). Maximum Urban Heat Island Intensity in Seoul. Journal of Applied Meteorology (41), 651-659.

Klysik, K. \& Fortuniak, K. (1999). Temporal and spatial characteristics of urban heat island of Lòdz, Poland. Atmospheric Environment (33), 3885-3895.

Kolokotroni, M. \& Giridharan, R. (2008). Urban heat island intensity in London: An investigation of the impact of physical characteristics on changes in outdoor air temperature during summer. Solar Energy (82), 986-998.

Lee, T., Lee, J. \& Wang, Z. (2012). Scaling of the urban heat island intensity using time-dependent energy balance. Urban Climate, 2, 16-24.

Lowry, W. (1977). Empirical estimation of urban effects on climate: a problem analysis. Journal of Applied Meteorology (36), 1377-1391.

Lui, W., Ji, C., Zhong, J., Jiang, X. \& Zheng, Z. (2007). Temporal characteristics of the Beijing urban heat island. Theoretical and Applied Climatology , 87, 213-221. 
Magee, N., Curtis, J. \& Wendler, G. (1999). The urban heat island effect at Fairbanks, Alaska. Theoretical and Applied Climatology. 64, 39-47.

Memon, R., Leung, Y. \& Chunho, L. (2008). A review on the generation, determination and mitigation of Urban Heat Island. Journal of Environmental Sciences (20), 120-128.

Molina, M. (2007). Efectos de los tipos de urbanización asociados al crecimiento urbano del área metropolitana de Santiago sobre la generación y comportamiento de micro islas de calor. Memoria de título Geografía, Universidad de Chile, Departamento de Geografía, Santiago.

Montávez, J., Rodríguez, A. \& Jiménez, J. (2000). A study of the urban heat island of Granada. International Journal of Climatology, 20, 899-911.

Moreno, M. (1993). Estudio del clima urbano de Barcelona: la isla de calor. Vilassar de Mar: Oikos-tau.

Oke, T. (1973). City size and the urban heat island. Atmospheric Environment (7), 769-779.

Salinas, F. (1982). Estudio experimental del efecto de isla calórica en la ciudad de Santiago. Memoria inédita para optar al título de ingeniero civil, Facultad de Ciencias Físicas y Matemáticas, Universidad de Chile.

Sarricolea, P., Aliste, E., Castro, P. \& Escobedo, C. (2008). Análisis de la máxima intensidad de la isla de calor urbana nocturna de la ciudad de Rancagua (Chile) y sus factores explicativos. Revista de Climatología (8), 71-84.

Sarricolea, P., Meseguer, O. \& Martín-Vide, J. (2011). Aplicación de la clasificación automática de Jenkinson y Collison a Chile Central. Conferencia Geográfica Regional de la Unión Geográfica Internacional (UGI). (p. 10). Santiago.

Yagüe, C., Zurita, E. \& Martínez, A. (1991). Statistical analysis of the Madrid urban heat island. Atmospheric Environment. B (25), 327-332. 